\title{
How to Improve Network Science: the Potential of (Empirically Calibrated and Validated) Agent-Based Modelling
}

\author{
Edmund Chattoe-Brown \\ School of Media Communication and Sociology \\ Bankfield House, 132 New Walk, Leicester, LE1 7JA, UK \\ ecb18@le.ac.uk \\ Simone Gabbriellini \\ Department of Economics and Management, University of Trento \\ simone.gabbriellini@unitn.it
}

\begin{abstract}
This article argues that the potential of Agent-Based Modelling (the capability for empirical justification of computer programmes representing social processes as dynamically unfolding individual cognition, action and interaction to reveal emerging aggregate outcomes) is not yet fully realised in the scientific study of social networks. By critically analysing several existing studies, it shows why the technique's distinctive methodology (involving empirical calibration and validation) is just as important to its scientific contribution as its novel technical capabilities. The article shows the advantages of Agent-Based Models following this methodology and distinguishes these clearly from the implications of apparently similar techniques (like actor-based approaches). The article also discusses the limitations of existing Agent-Based Modelling applied to social networks, enabling the approach to make a more effective contribution to Network Science in future.
\end{abstract}


Keywords: Agent-Based Modelling, Calibration, Validation, Rational Choice Theory, Dynamic Networks, Friendship.

\section{Introduction}

This article shows how the scientific potential of Agent-Based Modelling is not yet fully realised in Network Science (hereafter NS) and why realising that potential would be advantageous. In a nutshell, the reason is that while the technical capabilities of Agent-Based Modelling are widely recognised, the scientific implications of its distinctive methodology are not. The article begins by using an early application of Agent-Based Modelling to the rigorous study of social networks (Hummon, 2000) as an illustrative case study for the method and its empirical implications. This Agent-Based Model (hereafter ABM) has been replicated (to ensure the robustness of its results) and new findings are presented illustrating points relevant to subsequent argument. During this analysis, the methodology of AgentBased Modelling is introduced (particularly the key concepts of calibration and validation). These components of Agent-Based Modelling methodology allow simulations to undergo empirical testing in a distinctively different way from statistical analyses that fit their models to data. The second section of the article shows (using a typological review) that, despite the age of Hummon's article, the issues limiting the capacity of Agent-Based Modelling to realise its full scientific potential in NS still operate. In particular, the discussion of methodology clarifies why actor-based approaches (which are an important contribution to the formal analysis of networks) remain importantly distinct from Agent-Based Models (hereafter ABMs) despite their apparent similarities. The final section discusses existing and future research ensuring that Agent-Based Modelling can realise its full potential in NS.

\section{A Case Study of an ABM: The Rational Dynamic Evolution of Networks}


A known advantage of ABMs is their ability to represent explicitly both individual cognitive processes and their aggregate consequences. Hummon's article illustrates this point by presenting an $\mathrm{ABM}$ in which individuals are supposed to make rational decisions about changing their personal networks based on costs and benefits of tie maintenance. The ABM is able to show what aggregate network structure results assuming that populations of individuals make sequential decisions thus. (Needless to say, this does not imply that real individuals do make decisions rationally or sequentially.) Inspired by analytical work from Jackson \& Wolinsky (1996), Hummon assumes that it costs two agents something to maintain a tie and that the benefits of ties accrue from being connected (preferably via short paths) to all others in the network. (Thus costs accrue directly to those who maintain ties while benefits ensue indirectly for all those who can use them in their connectivity.) When each agent decides, Hummon assumes, they consider all possible personal networks making or breaking no more than one tie (which thus includes leaving the network unchanged) and choose to implement the utility maximising arrangement. The ABM then schedules this sequence of individual decisions and unfolds the resulting consequences for whole network at each time step (with the network state for the next individual to decide being the outcome of the decision by the last agent).

For an effective understanding regarding the potential of Agent-Based Modelling in the scientific analysis of networks, however, it is necessary to be clear that its advantage is not only the well-known general ability to represent individual cognition, action and interaction (and their aggregate consequences) explicitly, but also a systematic procedure to test these representations empirically in a distinctive way (without which one cannot reasonably expect ABMs to be used for reliable policy advice or prediction). The empirical realism of Hummon's particular assumptions has no bearing on the first advantage (but its relationship to the second will be discussed in detail shortly). The ABM could (as we will show) have 
represented different behavioural assumptions equally well. Thus while the technical achievement of an ABM is explicitly representing individual processes of decision/action and their aggregate consequences, its methodological accomplishment (far more clearly articulated since Hummon's article) is providing effective answers to two questions. Firstly, how do we (in fact) establish the way that individuals make decisions in some context and what values should be assigned to the resulting parameters? ${ }^{1}$ (This latter process is usually called calibration while the former has been referred to as specification.) Secondly, how do we establish whether the individual and contextual claims we make in the $A B M$ generate corresponding aggregate consequences to those observed empirically? (This process is generally called validation.) It is in addressing these questions, this article argues, that crucial differences between network analysis and Agent-Based Modelling approaches clarify sufficiently for the distinctive potential of the latter in NS to emerge.

Because Hummon's article is now 20 years old, the first stage in our argument has to be ground clearing. Although this article is mainly about the importance of calibration and validation in displaying the full scientific potential of Agent-Based Modelling, the importance of "debugging" (ensuring that an ABM actually does what its programmers intend) should not be forgotten. Thus, while there were practical advantages to reimplementing Hummon's ABM using modern software (ease and speed but also deeper understanding) there was also a methodological justification. Before considering the calibration and validation issues on which the arguments of this article hinge, we wanted to ensure that the ABM Hummon described definitely did behave as he reported and that its behaviour was not an artefact of the specific programme or programming language he chose.

\footnotetext{
${ }^{1}$ For purposes of comparison, we are obliged to follow Hummon's (tacit) assumption that no external environment bears on these networks (for example travel costs in interaction). This article therefore sets aside the (nonetheless important) issue of how we specify and calibrate environmental (non-agent) aspects of ABMs.
} 
Fortunately, part of Hummon's analysis provides an extremely challenging test for such a replication to meet. He presents an experimental design varying five parameters of his $A B M$ : Network size, costs of a tie, benefits of a tie, initial network density and social practices for making and breaking ties (pages 239-242). For each of the resulting experimental conditions, he ran the ABM 100 times to quantify stochasticity (the effect that "pure randomness" - as opposed to the specific ABM assumptions - has on outcomes). He then reported, for each combination of parameters, the modal network structure that resulted. ${ }^{2}$ This gives a data set of 17328 points. To replicate this, we recreated Hummon's experiment using our own ABM implementation and scored one for each combination of parameters where our $\mathrm{ABM}$ produced the same modal network structure reported by Hummon and zero otherwise. Doing this produced a match of $91 \%$. Given the large data set and information loss from only comparing modal structures, we consider this a robust replication. ${ }^{3}$

\footnotetext{
${ }^{2}$ Hummon (pages 234-235) identifies four basic network types: A null network without ties, a complete network with every node connected to all others, a star network in which all other nodes are only connected to one "hub" node and a ring or shared network in which all nodes connect to just two others. Sorted tie counts simply and clearly represent these types. For five nodes, for example, null is ( $\left(\begin{array}{llll}0 & 0 & 0 & 0\end{array}\right)$, complete is (4 4444$)$,

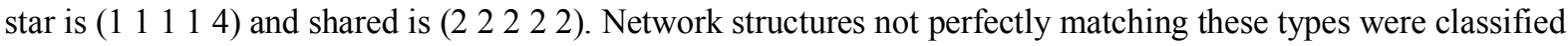
as near types. For example, the network structure $\left(\begin{array}{llll}1 & 1 & 2 & 2\end{array}\right)$ which we might call a chain is closer to a shared (Hamming Distance - sums of square of differences between corresponding positions - of 2) than to a star (Hamming Distance of 6) and is therefore classified as near shared. This procedure is always guaranteed to assign a network into one of only eight types (though the near types are obviously not homogeneous). Hummon does not discuss whether Hamming Distance "draws" are possible and, if so, how they are handled.

${ }^{3}$ This is the most austere possible replication test. Certain results should probably be allowed to raise the match score further. Firstly, Hummon himself identifies structures like near stars and some credit should be given where Hummon's modal structure is star and ours is something related like near star (rather than something very different like null). Secondly, because of stochastic effects, it is possible that in some runs, the modal type is only barely modal. In these cases, identifying another common type for the same parameter combination should
} 
Before going further and using our implementation of Hummon's ABM to present additional results and discuss their ramifications, it is necessary to consider the implications of a different aspect of Agent-Based Modelling methodology. Although the technical advantage of Agent-Based Modelling is explicitly representing individual behaviour and rigorously unfolding its aggregate consequences, this activity has to occur in an appropriate context to realise the method's full potential. In particular, the standard presentation of the methodology (Gilbert \& Troitzsch, 2005) argues that any ABM should represent a clearly demarcated social phenomenon. Hummon is unclear about the intended application of his ABM and this is consistent with similar lack of clarity in Rational Choice approaches generally. He writes: "Friendship ... can be conceived in terms of costs, etc. Similarly, sharing advice can be conceived in terms of costs and benefits ..." (page 221) Unfortunately the phrase "can be conceived" is about as scientifically non-committal as it can be. What are the arguments for this conception and on what evidence are they based? What alternative conceptions exist and what is the balance of evidence for those? While an instrumental element to advice sharing seems fairly plausible, treating friendship similarly seems open to reasonable objection. This issue is highly germane to the argument of the present article (for example in analysing concepts like the "preference for homophily" in actor-based approaches discussed subsequently). On one hand, we might take the Rational Choice approach as a descriptive (specification) claim. This is, in fact, how people decide. If this is so then it should be possible to elicit reports of decision making directly (using qualitative interviews for

probably be given some match credit. Hummon acknowledges the latter point (page 242). Unfortunately the colour images he provided as supporting materials (which show not just modal structure but the actual structure distribution) are now absent from the publisher web site where they are reportedly available and cannot be located by the current journal editors. 
example). While the content of such interviews should be intelligible ("Tell me why you broke contact with Bob") the idea that they could deliver cost and benefit data to calibrate a Hummon style ABM does not seem plausible. In fact, however, it doesn't seem that Rational Choice is usually intended to be an empirical statement about how people decide. Instead, the claim typically made is that its consequences are compatible with data. The difficulty with this position is that it isn't clear how persuasive it really is. Perhaps multiple individual level accounts are compatible with what is observed in aggregate. ${ }^{4}$ According to this presentation of Rational Choice, the aim is not to be descriptive and calibrate costs and benefits directly but merely to demonstrate compatibility with data using notions of costs and benefits as theoretical constructs endorsing the argument. This position can be seen as following the broadly statistical approach to data analysis. When we run a regression model evaluating the effect of class origins on educational success, for example, there is no aspiration to calibrate the slope and intercept independently. (Indeed it is not clear what that would mean.) Instead, the purpose of regression analysis is just to tell us, based on certain assumptions (for example that a linear relationship actually exists), how the data behaves relative to the model. Furthermore, while it tells us reliably about patterns in data, it is unclear what connection slope and intercept might have to causal processes (like parental support or antipathy/sympathy between classes ${ }^{5}$ ) presumably generating the observed pattern. ${ }^{6}$

\footnotetext{
${ }^{4}$ The technical term for this is equifinality (Beven \& Freer, 2001).

${ }^{5}$ The point here is not the trivial one that analysis with too few (or the wrong) variables will fail. Some causal processes are "interactional" (like class antipathy) or "adaptive" (like parental support) and may therefore not be capable of effective representation by variables at all.

${ }^{6}$ It is conceptually useful to distinguish the Data Generation Process (hereafter DGP, Hendry, 1995), the real world actions and interactions producing any data we happen to collect, and models attempting to characterise that data. Although many approaches hypothesise that the model is the DGP, this seems unjustifiable if the
} 
We can now provisionally state the methodological core of Agent-Based Modelling and differentiate it effectively from the statistical fitting approach although more argument is required to support the claims made and demonstrate their importance for the potential of Agent-Based Modelling in NS. An ABM is designed to represent a specific domain (so an $\mathrm{ABM}$ of friendship may well encapsulate different processes and parameters from an $\mathrm{ABM}$ of advice sharing and the differences should be empirically justified). The intention is that ABMs should definitely make realistic (albeit abstracted) claims about the social world. Thus cognitive processes and any associated parameters should at least in principle be accessible using appropriate research methods (surveys, qualitative interviews, laboratory experiments) and this is one reason why it must be clear what domain an $\mathrm{ABM}$ is intended to represent (so it is evident what specification and calibration data is relevant). The same logic applies to validation. If we know what the $\mathrm{ABM}$ is an $\mathrm{ABM}$ of then it is clear what data (friendship network data rather than advice sharing network data) will support or falsify it. This contrasts with a situation where behavioural hypotheses (Rational Choice) and associated parameters ("costs and benefits") are not established independently (specification) or measured (calibration) but are justified purely by compatibility of model outputs with data (fitting).

This distinction allows us to consider another point in our argument arising from Hummon's ABM. The present article is not intended to persuade people to take a fundamentally different view of NS. It intends only to make as clear as possible that there are different views to be taken and show that Agent-Based Modelling encapsulates an importantly different view that might be of distinctive value in the scientific understanding of social networks (particularly with respect to the way that data is used in evaluating models). Let us therefore suspend judgement about calibration "versus" fitting. Let us suppose we fully endorse Hummon's

modelling approach does not attempt to directly access or represent the underlying social process. Arguably, therefore, Agent-Based Modelling tests this hypothesis through combined calibration and validation. 
$\mathrm{ABM}$ of rational network formation. It still has to be the case that the aggregate properties of the simulated networks can display congruence with empirical networks (even statistical fitting requires this, let alone calibration and validation of an ABM). It therefore seems odd that Hummon barely mentions real data. The only claim (page 222) we can find is: "These structures [null, star, complete and perhaps ring networks] are common and often found in empirical studies of group interaction." Without any references, we struggle to evaluate this claim effectively but it seems almost constitutive of analysing empirical relations that two of Hummon's structures (completely connected and null) are not common (being almost the antithesis of what we mean by social networks). ${ }^{7}$ We are aware of a fairly well known research strand initiated by Bavelas (1950) involving network communication in small groups but examining the original articles offers no empirical justification for the star structure which was imposed experimentally.

A related difficulty has already been raised. Because research into social networks is empirically strong, one systematic way to refute Hummon's (unsupported) claim would be to take a sample of research, see what network structures it reports and how many of them are broadly null, star or complete. But what constitutes a fair sample? If we take Hummon at his own word and say that his ABM represents friendship networks then we find a large body of classroom sociometry (for example) in which null, star and complete networks are not observed. See, for example, Forsyth and Katz (1946), Moreno et al. (1943) and Potashin (1946). The same applies to advice sharing networks. See, for example, Yang and Tang (2004). Of course, these examples are hardly systematic but since Hummon offers no evidence at all, perhaps they provisionally establish the burden of proof against his assertion. This is a good point in the argument to illustrate the potential weakness of an ABM that (like Hummon's) is neither calibrated nor validated (and thus move beyond the mere

\footnotetext{
${ }^{7}$ Social networks often contain completely connected sub networks (i. e. cliques) but that is a different claim.
} 
representational flexibility of Agent-Based Modelling to its distinctive methodology and relationship with data). This weakness arises from the very large number of ABMs no less implausible than the one presented which, nonetheless, give substantively different results. This weakness can be viewed either from the empirical perspective or from the standpoint of an actual working ABM. We shall present examples of both perspectives. From the empirical angle, it is unclear why agents should only consider breaking or making single ties in each decision and why they would consider all possible combinations of such changes. (An unlikely behavioural consequence of this, for example, is that people in bigger networks have to think faster!) In fact, one of Hummon's illustrations of his model seems to depend specifically on this assumption. Looking at Figure 4 on page 233 , we see the final state of a small network. It is the final state because no agent can change any single tie and improve its personal utility. At the same time, we know from Jackson and Wolinsky's analytical results that this structure is not optimal for the corresponding parameter values. The optimal structure is a star. If, however, agent 2 were able to break a tie to agent 5 and make one to agent 1 simultaneously then the optimal structure could be achieved. It is thus only the absence of a path via single tie changes that keeps the network suboptimal (and this is a limitation imposed by an empirically ungrounded assumption). This outcome is thus extremely sensitive to the particular non-empirical assumption made. While it is clearly behaviourally implausible that agents consider all combinations of making and breaking $n$ ties (the number of alternatives to consider would explode) it is somewhat plausible (or at least no less plausible than Hummon's assumption) that agents would consider a much smaller number of alternatives (say 2 or 3 ) for each decision involving slightly larger numbers of ties made and broken. ${ }^{8}$ It is an interesting question (which Agent-Based

\footnotetext{
${ }^{8}$ There is another tacit assumption in Hummon's model that is relevant here. Presumably the utility of connection implies some sort of access to benefits like information or resources flowing through the network.
} 
Modelling is eminently suited to investigate) whether such a decision making approach would be less susceptible to suboptimal equilibria. (ABMs could also investigate whole other classes of decision process like imitating tie structures of "successful" agents.) Another example where one can follow the logic of Hummon's assumptions differently (but no less plausibly) involves the social practices associated with tie making and tie breaking. Hummon distinguishes between unilateral action (which ignores the situation of the other agent) and mutual action (which doesn't). Unfortunately, there is an underlying issue with coherent behavioral interpretations of tie making and breaking. If you are truly selfish (the argument runs) then all your actions should be unilateral. But following the argument further, this might actually be counter-productive even in selfish terms. If, by deleting a tie with another agent, you lower its utility then its immediate reaction may be to remake the tie, thus rendering your action pointless (and in a situation with limited decision opportunities, wasteful). This, presumably, suggests the idea of mutual tie formation and deletion, which only occurs if you don't worsen the position of the other agent. Unfortunately, this approach doesn't seem to follow the logic of the situation through either. If I do something that worsens your situation, you can only undo it next time you get to make a decision (meaning I get the benefit of breaking the tie for a while) and even then, with everything else changing, you may not remake the tie because doing something else may gain you more utility (in which case I may continue to benefit from breaking the tie). Thus it is not necessarily futile, as it might at first appear, to make or break a tie worsening the situation of another agent. Given the very high levels of knowledge agents are supposed to have about costs, benefits and the state of the network, it seems no less plausible than Hummon's assumptions that an agent might decide to make or break a tie only if, given the current state of the network,

This being so, agents could also take account of how often a particular tie gave access to such resources in practice and this might influence the decision to maintain or break one tie rather than another. 
immediately undoing the change would not be the top priority of the other agent. Each agent is assumed to know so much about the network already that reasoning about others is barely harder than reasoning about oneself!

Another way to understand ABMs (and the limitations of not calibrating and validating them) better is thus constructing variants with different plausible assumptions. For example, to what extent is the small and sharply defined set of network structures discussed by Hummon dependent on the (arbitrary) fact that the Jackson and Wolinsky utility function penalises longer paths between agents very heavily. Because costs and benefits are assumed to be greater than zero and less than one and benefits are raised to the power of the path length, a benefit of 0.5 has already been reduced by half if the path length is two rather than one and by half again if the path length is three. This may have the effect (not too surprising ex post even without analysing an $\mathrm{ABM}$ ) of enforcing a star structure as the only one achieving total connectedness through very short paths. Hummon doesn't justify this utility function but (depending on the specific social domain being modelled) it seems potentially either too harsh or too generous. For a gossip network it seems unlikely that, in order to benefit, you would have to be extremely close to the source (it being in the nature of gossip to spread). By contrast it seems unlikely that advice would spread successfully through even a single intermediary. Hummon assumes fixed networks but we suspect that advice works substantially by dynamic referral (see, for example, McGrath et al., 2003) rather than message retransmission: "You need to talk to Mary. Say Joan sent you." Be all that as it may, however, we find that when we penalise longer paths less, the behavior of the model changes substantially (regardless of other parameters like the initial density levels, the method used to add/remove links and the network size). For example, there are a lot more complete networks for combinations of parameter values that previously yielded stars. Further, the predominance of near stars in 4 and 5 node networks is drastically reduced. Taken as a whole, the system 
basically divides between complete and null networks with only a narrow combination of parameters for which stars and near stars are the most likely outcome.

It is important to interpret this discussion carefully. This outcome isn't "better" than Hummon's (since it is no more calibrated or validated). The problem is that (on the same grounds) it isn't worse either. The point is that, without calibration or validation, Hummon's specific "result" evaporates if one happens to make different arbitrary (but plausible) assumptions. It is not clear how this situation can lead to progressive improvement in the quality of models. This proliferation of "not implausible" assumptions thus forces us towards calibration or validation (and ideally both) to distinguish the many models that are merely plausible from the (presumably) far fewer that are scientifically supported by evidence. ${ }^{9}$ Only ABMs matching real data in validation serve as even possible candidates for social explanation (since statistical fitting models do no worse and often with greater parsimony) but, of those, only the subset with behaviour and parameter values independently corresponding to data (calibration) are really credible candidates (since the possibilities for fitting a complicated ABM with many "free" parameters are just too expansive.)

Before moving on we provide another example of analysing plausible variant assumptions in ABMs. We know from previous research (for example McPherson et al. 2006) that individuals do not have large numbers of ties unless they are almost costless (as on Facebook). As such, we have independent reasons for worrying about the implicit assumption in Hummon's ABM that there are no constraints on the numbers of ties that any individual can maintain. When we impose a constraint on tie capacity for each agent, we find that complete networks almost disappear. For 3-node networks, the dominant structure is now

\footnotetext{
${ }^{9}$ Rhetorical issues also arise. Most models justify what they include but excluded behaviours are not discussed. Thus the overall plausibility of a models depends on the reader also noticing what is absent. This means that an ABM that appears plausible in isolation may look increasingly arbitrary when compared to others.
} 
near shared. For 4-node networks, the only outcomes are near star and null while for 5 and 10-node networks we observe near shared and null networks. As before, therefore, Hummon's results are largely undermined simply by replacing one "not unreasonable" assumption with another (and we have no scientific way to choose between the variants).

\section{A Typological Review of ABMs Involving Social Networks: The Difficulties Remain}

We now face something of a challenge in advancing our argument. The difference between the fitting and calibration/validation approaches is neither simple to explain nor self-evident from published research. Therefore discussing an example in detail was necessary to present the distinction as clearly as possible. For practical reasons, a simple example also makes this task much easier. However, a critic could reasonably ask whether the issues arising in Hummon are typical of Agent-Based Modelling applications to social networks and, in particular, whether these difficulties simply reflect the age and ground breaking nature of his contribution. Perhaps Agent-Based Modelling has simply raised its game empirically in the last two decades? The difficulty is then to strike a balance between an analysis of all ABMs involving social networks (which would be impossibly onerous) and an unsupported claim that issues we raised using Hummon's article are still typical.

The compromise we arrived at was to identify all articles in the online versions of Social Networks and Network Science containing the search term <agent-based> (as of 09.06.20) and categorise them according to calibration and validation status. ${ }^{10}$ Exemplars in each category will be discussed in more detail as appropriate to the argument. (The full list of

\footnotetext{
${ }^{10}$ The reasons for looking at each of these journals were somewhat different. Social Networks is a much older journal (and pre-eminent in network analysis) and thus provides a sample of more convincing size. Network Science, while newer, has a different emphasis (for example explicitly mentioning computational approaches in the scope of potential submissions). Between the two of them, it would be hard to argue that any conclusions about the use of ABMs in modelling networks were not both generalisable and up to date.
} 
articles and their category assignments is available from the first author on request.) The argument starts with a more synoptic analysis of the relatively large number of articles in Social Networks and then proceeds to confirmatory scrutiny of the much smaller number of cases in Network Science.

The total sample size of the search for Social Networks was 182. 4 search hits were rejected immediately as not being research articles (3 book reviews and 1 file of supplementary material). Since Hummon has already been discussed in detail, this brings the remaining number of cases for analysis down to 177 . By far the biggest category was false positives (132) that, on closer inspection, didn't contain the search term after all, reducing the effective sample to 45. A further 24 articles mentioned ABMs incidentally (only in references for example) but these citations were no substantive part of their argument. This left only 21 articles that either clearly involved ABMs or couldn't be excluded from doing so without full reading. This close reading eliminated 5 further articles from the sample. This left a final sample of 16 articles, rendering Agent-Based Modelling a rare approach in the analysis of social networks generally (and thus supporting the value of the present article). Of these, 8 articles were neither calibrated nor validated. Such ABMs thus face the same problems already discussed in the context of Hummon. Without calibration it is not possible to establish what social phenomenon (if any) they supposedly apply to. Without validation, there is no way of assessing whether they do, in fact, have any scientific relevance. In the final analysis only 7 articles out of 177 - a mere 4\% (Arentze et al., 2013; Bravo et al., 2012; Growiec et al., 2018; Iribarren and Moro, 2011; Snijders et al., 2010; Starnini et al., 2016; Ziegler 2008) were definitely validated (in that they presented comparisons between real and simulated data such that the degree of match between these could be evaluated by the reader). We will discuss these 7 articles in an arbitrary order that most clearly develops our argument. 
Before doing this, however, we will discuss an article (by Stadtfeld et al., 2020) which falls into a unique empirical category. This discussion again shows how the methodology of Agent-Based Modelling (using concepts of calibration and validation) is an effective organising principle for understanding the scientific strengths and weaknesses of ABMs with a significant network component. Stadtfeld et al. make the important point that disliking as well as liking ought to shape the structure of networks, particularly in the context of explaining group boundaries. They add "disliking mechanisms" to the standard software for statistical fitting of dynamic networks (RSiena) and show improved fit for real longitudinal classroom data. Thus far, the article would not meet the criteria for inclusion in our sample. However, in the second part of the article, they report using the statistical software "as an ABM" (see the discussion of Snijders et al. 2010 below for concerns about this conflation) and "run it forward" (using the same parameter values that achieved best fit on the classroom data) to see whether variants with or without disliking create more plausible looking networks and conclude that, again, the latter contribute to improved model performance. So, this model is calibrated (in a special sense) but not validated. Such models are unusual for a reason that can easily be explained using standard Agent-Based Modelling methodology. While applying the parameter values from real data could be seen as more rigorous strategy, without validation, we cannot tell if it has actually been successful. Furthermore, if it turned out to be unsuccessful we couldn't be sure if this was a result of the parameter values (which are fitted statistically and not independently calibrated) or of the empirically questionable assumptions incorporated into the statistical software (which will be further discussed shortly). Stadtfeld et al. freely admit that the resulting networks from the simulation exercise do not look empirically convincing but don't discuss the potential role of validation at all. Having analysed this unique case, we now turn to a similar methodological analysis of the small number of ABMs that are (at a minimum) validated. 
Ziegler's (2008) ABM was, because of its application area (the historical anthropological phenomenon of the Kula ring involving ceremonial goods exchange between island traders), almost impossible to calibrate. (It also created problems with validation as the ethnographic sources do not agree on the actual trading network structure.) Ziegler's analysis thus involved implementing a theoretical account of kula (using game theory). As such, the fact that the fit between simulated and real data was (by the author's own admission) rather poor may say more about the inapplicability of the theory than it does about the Agent-Based Modelling approach per se. Had direct ethnographic information about trader behaviour been available, it is at least possible that an $\mathrm{ABM}$ implementing it would have shown greater similarities to the observed trading network. Nonetheless, Ziegler's work (attending carefully to data) is more likely, for reasons already explained, to contribute to progressive research using ABMs than examples that are neither calibrated nor validated.

The article by Iribarren and Moro (2011) does not face the problem that the data needed for calibration are no longer available but well illustrates the limitations of ABMs that are only validated. This research starts from data on email propagation and identifies a distinctive pattern of transmission through networks. An ABM is then constructed which, to some extent, reproduces this pattern. But without calibration, we cannot tell if the reproduction occurs because the assumed micro-process is what gives rise to the observed communication patterns or whether the match between the real and simulated data is fortuitous. Furthermore, we have no way of telling how many other somewhat plausible variant ABMs might have matched the data equally well. ${ }^{11}$ (The less precise the observed fit, the greater the presumed

\footnotetext{
${ }^{11}$ The possibility of complexity creates another difficulty. Casual inferences about the micro behaviours leading to particular aggregate patterns (and vice versa) can simply be mistaken when the nature of the system creates non-linearity or emergence. This is well illustrated by the famous residential segregation model (Schelling 1971) in which a minority preference for one's own kind can still lead to clustering while a strong majority preference leads to an absence of clusters.
} 
likelihood that variant assumptions or, more worryingly, a completely different ABM might achieve it.) Nonetheless, as with Ziegler's article, an ABM that matches some aspects of what we know makes more of a contribution to progressive research than one that does not even attempt this (though any attempt at calibration, however tentative, would obviously be better still for reasons already discussed.)

To emphasise again that its methodology is an important organising principle for analysing ABMs, exactly the same argument applies to Starnini et al. (2016) despite its very different topic. In this case the domain is organisational social interaction (in hospitals and schools) and validation uses various detailed measures of these interactions. In the ABM, however, agents are assumed to move randomly and select partners based on "attractiveness". Not only are these assumptions implausible (and attractiveness, like costs and benefits, might prove hard to calibrate even in principle) but the fact that the ABM seems to explain settings as diverse as academic conferences and hospitals, far from being encouraging, could actually be problematic from the perspective of Agent-Based Modelling methodology. To return to the earlier point about ABMs being constructed to represent specific social domains, if a model of random mixing and "attractiveness" works at a conference drinks party then it really shouldn't work somewhere as different as a hospital (where people have time and location structured jobs to do and many social interactions are prescribed by these - operations, case meetings and ward rounds for example). If the $\mathrm{ABM}$ can match data from diverse organisations with such implausible assumptions then it may just mean that the level of match achieved is insufficiently challenging and thus that many other sets of assumptions could have realised it too (which would be unhelpful).

Another useful example illustrating and reiterating the interplay of several arguments already raised is provided by Growiec et al. (2018). Drawing extensively on theory about social networks, social capital, trust and economic performance, they specify an ABM which is 
validated on correlations in model output and corresponding survey data. However, this approach faces five challenges within the logic of Agent-Based Modelling methodology. Firstly, they show this match for two parameter combinations without explaining why these were selected. Secondly, there is no objective basis for deciding how meaningful the correlation matches are. For example, if these values differ by as much as 0.16 (in the range $0-1$ i. e. about $16 \%$ ) then is this a good match, a moderate match or even (possibly) a weak match? The reader is left to "eyeball" the various numbers and draw their own subjective conclusions. (This should be compared with the situation in traditional statistical analysis where the likelihood of an outcome can be established relative to a relevant null hypothesis.) Thirdly, because the authors assume that the model social network is generated according to the Watts-Strogatz "small world" algorithm (Watts and Strogatz, 1998), they are forced to admit that two key parameters whose variation they explore extensively simply cannot be calibrated using survey data. (And the possibility remains open that the real network is not so generated - a specification issue - in which case these "parameters" have no empirical referent at all.) They argue that since these parameter values are conceptually bounded (i. e. $0-1)$ they can simply report the effects of exploring the whole parameter space. This is true as far as model behaviour goes but does not tell us what specific parameter values we should use for genuine validation. Fifthly, although the authors attempt to compare the model to other survey data, they admit that they often have to use whatever data exists rather than what the model actually calls for. Thus any appearance of correspondence reported is dependent on the unsupported claim that the available data is an effective proxy for the data needed (which is a "fitting" style of argument rather than an independent calibration). Finally, as already suggested, while extensive justification is provided for what is included in the model, what is excluded is not justified. For example, if the model appears to fit data but assumes static networks, absence of reputational processes and the ability to represent all forms of economic 
collaboration as a simple Prisoner's Dilemma then might the reader worry that the apparent validation is fortuitous or that the data concerned simply can't discriminate adequately between a wide variety of plausible models. Overall, the reader is left very clear that there is some sort of reported congruence between real and simulated data (though not necessarily how impressive that is) but very unclear about the extent to which that congruence is based on a systematic process of calibration (or more general empirical "grounding" of model assumptions). If the model is complicated enough (and has sufficient "free" parameters) and the data is general enough (a single correlation is much less discriminating than a time series for example), then maybe some measure of match can always be achieved?

The article by Bravo et al. (2012) raises slightly different methodological issues but nonetheless gives further weight to the argument developed here. This article is based on relatively unusual data (game theoretic laboratory experiments) and also uses an ABM in a non-standard (but still important) way. The argument proceeds in two stages. In the first, an $\mathrm{ABM}$ is validated on experimental data. The authors report that the ABM "exactly replicated" (page 484) the experimental data but it is hard to reconcile this claim with the plots of investments and returns shown on pages 484-485. There is clearly a subjectively credible degree of match but an important challenge still remains for Agent-Based Modelling to adopt or develop tools for characterising (and most importantly reporting) the exact level of match and what it signifies. In any event, the usual problem regarding validation without independent calibration arises here. This is certainly a model approximating the data, but what scientific reasons would we have for preferring it over other such models? However, part of the reason for building the ABM (which, whatever its limitations, is still more rigorous than one that is not validated) is to conduct experiments that are impractical in the laboratory. For example, if experiments are done with 10 subjects, how does the system behave with 100 ? This is a useful question (particularly regarding social networks) and a 
valuable application of an $\mathrm{ABM}$ but an obvious problem presents itself. If the $\mathrm{ABM}$ effectively reproduces the decision processes and interactions of individuals (i. e. it is specified and calibrated) then it should scale and give sensible answers for 100 agents (though circumstances mean that we cannot really check this) but if the apparent validation is merely a fortuitous association (in the absence of specification and calibration), then it may not scale after all. Again, this analysis shows how methodology (and research design) are important organising principles for effective empirical ABMs.

We have saved the trickiest (and potentially most controversial) cases until last. Recalling the point we made about the aim of this article not being to show that different methods are right or wrong but merely that Agent-Based Modelling has unexhausted potential to analyse networks scientifically, we nonetheless have to problematise a particular strand of research that (without criticising its legitimacy) has the potential to obscure the important contribution which ABMs can make. We have also saved this discussion till last because it relies on having a thorough understanding of how ABMs work, what calibration and validation involve in the Agent-Based Modelling context, how these are distinct from fitting (validation without calibration) and what potential weaknesses arise when the Agent-Based Modelling methodology is not fully implemented. (As we have seen most ABMs dealing with networks in our sample were neither calibrated nor validated and while a few were validated, we couldn't find a single example to discuss in the whole set of articles whose calibration was unambiguously systematic). ${ }^{12}$

\footnotetext{
${ }^{12}$ Abdou and Gilbert (2009) and Hägerstrand (1965) provide just two really good examples showing that the full Agent-Based Modelling methodology is both feasible and effective.
} 
The research in question is the stochastic actor-based modelling proposed in Snijders et al. (2010) to analyse dynamic networks. ${ }^{13}$ The first point is a purely semantic one. In the sample examined, there are several authors who believe (despite Snijders et al. using distinctive terminology) that agent-based and actor-based approaches are equivalent. For example, Simpson (2016) writes: "Hypotheses are tested using Stochastic Actor-Oriented Models ... a type of agent-based model for the statistical inference of longitudinal network dynamics ..." It may seem pedantic to make this very specific textual point but the whole argument of our article hinges on exactly why and how these approaches are not the same and why it matters. Having discussed Hummon in detail and a number of other ABMs involving networks more briefly, it is now much easier to explain what the issues are that separate actor-based and agent-based approaches (without attempting to adjudicate between them). Although statistically elegant, the actor-based models are not traditionally statistical (in the sense of fitting relatively simple equations to data) because they have to deal with agency in networks and dynamic networks at that. It is thus unsurprising (and this approach is actually much more generally used in solving challenging statistical problems) that simulation rather than

\footnotetext{
${ }^{13}$ Arentze et al. (2013) is an impressive but problematic case. Firstly, it is unclear if it is intended to be descriptive or to solve an instrumental problem (generating plausible network structures for other uses.) Secondly, it is based on the conflation between actor-based and agent-based approaches which we argue here is mistaken. Thirdly, although it is arguably the nearest the sample has to a calibrated and validated ABM, the calibration occurs on an abstract theoretical model of utility maximisation. While it is impressive that a calibrated version of a theoretical model does match aggregate network properties fairly well, it is less clear how this should be interpreted. The idea that all relationships are explicable by a standard calculus of utility (with a "utility of homophily" for example) seems sufficiently implausible that the fact that the calibrated model reproduces the data seems unlikely to be causal. Perhaps the real data (which all involves relatively smooth distributions) is just too easy to match, particularly if the model still contains "adjustable" parameters (is not "fully" calibrated). This returns us to earlier concerns about whether utility models can be calibrated and (if not) how challenging a test it actually is to fit them to particular patterns of data.
} 
estimation is the core of the approach. In a purely technical sense, we could say that actorbased and agent-based models are equivalent because both specify processes, originating at the micro level, that then give rise (through computation) to aggregate phenomena that can be compared with equivalent real data (in this case network structures). However, they also differ in several crucial areas that can be understood effectively through the Agent-Based Modelling methodology already discussed. The first of these is whether or not the approach only has an aspiration to validation or to independent calibration as well. As already suggested, the logic of Agent-Based Modelling methodology commits it to the latter (even though published examples do not always deliver on that commitment as we have seen), but for actor-based approaches the situation is less clear-cut. On one hand, actor-based models do make explicit claims about the situation of actors in networks, just as Hummon did, and this clarity is a benefit of the approach almost regardless of whether these claims are true (just as the programmatic importance of the Hummon $\mathrm{ABM}$ is much greater than the quality of its assumptions or outcomes would suggest). However, it is much less clear whether these claims are presented as empirical. Reading Snijders et al. (2010) regularly gives the impression that achieving fit is the dominant goal and that the empirical status of assumptions is not a major consideration. For example, they write (page 48) "The choice between these representations of transitivity may depend both on the degree to which the representation is theoretically convincing, and on what gives the best fit" and later (also on page 48): "Therefore these two effects will be difficult, or impossible, to distinguish empirically, and the choice between them will have to made on theoretical grounds." From an Agent-Based Modelling perspective, the status of both claims could be questioned. We have already suggested why fit alone is not enough because of the potential for equifinality and it is not clear what (if anything) it means for a description of a social process to be "theoretically convincing" independent of its empirical justification. It seems to us that saying that two 
theoretical or statistical effects cannot be distinguished empirically ought to sound warning bells for their scientific status.

If we do not feel able to accept theoretical or technical justifications for the claims encapsulated in actor-based models, we can ask all the same questions (and for the same reasons) as we do about ABMs. For example, Snijders et al. say (2010): "It is assumed formally that actors have full information about the network and the other actors." Is this assumption in fact empirically likely and what reason(s) do we have for supposing it to be true ${ }^{14}$ It is here that we can first see two important and related divergences between the approaches. In Agent-Based Modelling, specification and calibration are about clearly establishing grounds for particular assumptions regarding individual cognition, action and interaction. These arguments should usually have an empirical basis, however weak and provisional. ${ }^{15}$ By contrast, in actor-based models these assumptions also seem to have a technical (but sometimes referred to as theoretical) dimension: If we don't assume this (or something like it) then the approach won't work. ${ }^{16}$ This situation is very common in formal methods and, understanding their motivation, it is unproductive to argue with technical assumptions on empirical grounds. We only remark that being obliged to make non-empirical assumptions could just as easily been seen as a weakness rather than a strength of formal approaches and that $\mathrm{ABMs}$ are not obliged to make such assumptions for analytical tractability because they are not intended to be analytical but process based. (Barring programming bugs, an ABM will unfold a dynamic process whatever assumptions it may

\footnotetext{
${ }^{14}$ In fairness, Snijders et al. immediately qualify this assumption but via further assumptions not empirically.

${ }^{15} \mathrm{~A}$ further problem is that there is no reason to suppose that existing data will be in forms suitable for direct use by Agent-Based Modelling as a novel method. The challenge is to identify what the problems are and how the data would need to be different without merely making excuses for Agent-Based Modelling.

${ }^{16}$ Compare what Snijders et al. (2010) have to say about the assumption of Markovian network change and what Bearman et al. (2004) say about it from "outside" the community of network researchers.
} 
encapsulate.) The second aspect of divergence is the degree of commitment to behaviourally grounded assumptions about social process. In an ABM, at least in principle, a model of evolving friendships would explicitly represent places where people could meet, what social practices influenced making contact (when you may comfortably speak to strangers for example), what would happen if the initial contact was soon (or not so soon) repeated and so on. Ideally, these social process descriptions would be based on the subjective accounts of individuals and avoid encapsulating theoretical elements without some empirical support. (Since these are no longer needed for technical reasons in an ABM, it isn't clear what other justification they have.) By contrast (and this is unfortunately unavoidably a matter of interpretation) it seems to be the case that actor-based approaches do incorporate weakly supported theoretical constructs into what they represent as process descriptions of social actors. Two examples will support this claim. It is unarguable that triads in networks are a descriptive fact but it is arguable, it seems to us, whether this descriptive fact arises causally from a corresponding "mechanism" of triad formation, often expressed in terms of probabilities. This seems to us potentially to mistake cause and effect or calibration and validation. From an Agent-Based Modelling perspective, the formation of triads (and other groupings like cliques) seems likely to arise from various kinds of socially organised colocation or communication: A knows B and C and they finally meet because A invites them both to the pub after work or $\mathrm{B}$ and $\mathrm{C}$ meet because $\mathrm{A}$ recommends $\mathrm{B}$ to $\mathrm{C}$ as having some skill or resource. But what we know about socially organised co-location suggests that three unit groups are actually relatively unusual. We even use the disparaging term "gooseberry" to refer to a single person in the company of a couple (with the preferred option apparently being one couple or two). In these terms then, what we want to know is why the pattern of social organisation we actually find (what, following Feld 1981, we might call foci of various sizes and based on different kinds of roles or group memberships) happens to generate the 
number and kind of triads we observe but without assuming that these must come from a dedicated mechanism that specifically causes triads (rather than triads being just one measured aspect of a more general social process). ${ }^{17}$ Seen like this, and the shift in perspective is small but vital, studying such ABMs might draw our attention to other distinctive aspects of friendship network evolution (possibly involving larger or more idiosyncratic sub network structures) that turn out to be no less important structurally but to which we have not previously been "theoretically primed" as we have with triads. ${ }^{18}$ The point here is not to claim that such probabilistic accounts of triad completion fitted to data are wrong (because by their own lights they are legitimate, in particular being part of a long established statistical tradition of model fitting) but only to explain why Agent-Based Modelling neither needs (nor actually wants) to follow this logic and why the logic they do (or at least ought to) follow offers distinctive advantages. Another example is provided by homophily. Again, this phenomenon is indisputably an empirical fact but (it seems to us) the causal and empirical status of a "preference" for homophily (Snijders et al. 2010) as an unspecified and uncalibrated self-contained mechanism to produce it is not indisputable. We can easily distinguish in processual terms (and thus implement in an ABM) beliefs or

\footnotetext{
${ }^{17}$ This illustrates the earlier point about the utility of the DGP concept. The model is not the system.

${ }^{18}$ This can be seen as a more general concern with actor-based approaches. The quality of fit is determined not just by selecting the right social process elements but by the elements you need to select for a particular network being available in the software. If it turned out that, somehow, 2 triads joined at one node (or 8 nodes in a circle) had a vital structural role in the future evolution of networks then not only would we miss this causal element (and thus presumably fail to model the network effectively) because there did not yet happen to be any theory of the effect but even if we somehow came up with the idea, the software might not support it. The danger is then that actor-based approaches become a self-fulfilling prophecy as regards the current state of theory in network analysis (this is the best fit we can achieve on the presumption that we already know most of what we need to about micro processes of network dynamics) rather than a tool for actually discovering and exploring causally important patterns in data.
} 
practices that directly exclude heterophily ("As a white woman in the American South of 1935, I would never voluntarily talk to a black man") and those that may, after the fact, leave behind an apparent residue of descriptive homophily. (For example, people may form relationships in an egalitarian fashion but these relationships would be more likely to fail over time if the protagonists have less in common culturally, are subject to external moral censure and so on.) As with the triad case, the issue arises not in the patterns of data but in what is considered to count as a behaviourally (rather than statistically or theoretically) grounded micro-level process account of that data and where the evidence for that process account comes from. In Agent-Based Modelling the tendency (however weak and imperfect currently) is towards direct empirical justification while in actor-based approaches the tendency seems to be towards existing theory, technical considerations and satisfying the goal of fit. ${ }^{19}$ Saying that social actors have a preference for the level of homophily we actually observe does not seem to be something that can be checked independently and its explanatory status can therefore be questioned. ${ }^{20}$

\footnotetext{
${ }^{19}$ One could almost say that the aspiration of Agent-Based Modelling is that calibration should not be done to enhance fit but for its own sake. Fit (as Bertrand Russell is reputed to have said of happiness) should not be an end in itself but a byproduct of doing something else (in this case learning about the decisions/actions of actors and their outcomes in the environment).

${ }^{20}$ There are also more subtle and interesting questions about homophily which could be explored by ABMs which we have not seen existing approaches draw attention to. Does homophily in one dimension have the possibility to compensate for its absence in others? (By rights, I should have almost nothing in common with Joe who is black, working class and barely finished school. Yet our shared enthusiasm for Hitchcock films forms the main basis of our continued association.) Do people segment the needs for similarity created by their attributes to create different kinds of friendships? I am fine not to talk obsessively about pop music with most of my friends because I have an outlet with some other enthusiasts. My genuine friendship with Mary only survives because we have tacitly agreed (after some very uncomfortable early experiences) never to discuss
} 
It is difficult (and possibly contentious) to try and account for such divergences in approach but they might be explained by such issues as methods or theories having undue influence on the way we see the world (or data), limitations that are recognised but tolerated on the grounds that there don't seem to be any viable alternatives, coincidental neglect of kinds of data (for example qualitative research into subjective accounts) that might challenge the prevailing view and so on. As we shall show in the last section, the distinctiveness of AgentBased Modelling casts new light on all of these issues.

Finally, we know from our earlier discussions of Agent-Based Modelling what the general problems are with validation only approaches (of which we can now see actor-based approaches as a slightly non-standard example). It may be that there is more than one actorbased model that can achieve any given level of fit. (It is even possible that different models with contradictory assumptions might do so which would clearly be problematic.) It is possible that fit is merely associative rather than causal and the difficulty in collecting (and consequent rarity of) longitudinal data with many waves may make really strong out-ofsample tests unfeasible. For any given level of fit, the behaviour of the system may be rather sensitive to assumptions encapsulated in the software that are no more than somewhat plausible. As with the Hummon ABM, different somewhat plausible assumptions might lead to importantly divergent outcomes. ${ }^{21}$

Despite problematising actor-based approaches in this way, the aim is not to imply that they are flawed or don't do what they purport (or even that what they do is not worth doing). Instead, it is to clarify the fact that ABMs do something different and that this difference religion on which we disagree violently. Again, the statistical view of homophily is at risk of concealing or foreclosing important process insights with at least some face validity.

${ }^{21}$ This is another area where lack of opportunities to challenge the results outside the capabilities of the software might be problematic. Unlike a typical ABM (Will 2009), it would be hard to "replicate" the Siena software (Ripley and Snijders 2009) and test the robustness of its results to the assumptions "under the hood". 
(with an effective and coherent methodology behind it) can make a difference to the way we conceptualise networks and research their evolution according to scientific criteria. ${ }^{22}$

Although the journal Social Networks is pre-eminent in research categorised as Social Network Analysis (and is also old enough to provide a generalisable sample), it is clear that the distinctive approach of NS means that it would be illegitimate to generalise from the former journal to Network Science. However, an "out-of-sample" design for a typological review of two different journals (however informal) has additional advantages. Having done an analysis and drawn conclusions from a larger and more temporally extended sample we are now much better placed to "test" these by a more focused analysis of the smaller number of cases of ABMs found in Network Science. It is to this analysis that we now proceed. To the present $(09.06 .20)$, there are only three instances of the search term <agent-based $>$ in Network Science (Génois et al., 2015; Henry et al., 2016; Sullivan et al., 2015). ${ }^{23}$

Encouragingly for our argument, two articles immediately fall into categories we have already analysed (and the third very nearly does). Henry et al. (2016) is another ABM that is neither calibrated nor validated and thus suffers from the same difficulties as those already discussed. Génois et al. (2015) is another ABM that is calibrated and not validated (like

${ }^{22}$ One very informal but concise way to put the issue with actor-based approaches might be: If you are going to invest so much effort and technical ingenuity in something that ends up being so nearly an ABM, why not go all the way and gain all the benefits (and avoid all the problems) that follow from the associated methodology involving specification and calibration as well as validation?

${ }^{23}$ There is a judgment call connected with the sole use of this search term. More recently other terms (like Individual-Based Modelling and Computational Social Science) have proliferated. Even though searching for $<$ agent-based $>$ may exclude research with similar content but different "labelling" it has the advantage of being fully comparable over the whole period of analysis. These alternative terms had not been invented in the early days of Social Networks. In our experience, there is nothing to suggest that research labelled as IBM and CSS is substantially different in the respects we are analysing here (and in any event the samples concerned would be too small to be useful for comparison). 
Stadtfeld et al., 2020 above). As such, while calibration apparently introduces rigour, without validation we still cannot tell if the ABM described is actually justified empirically.

By contrast, Sullivan et al. (2015) introduces another distinctive issue for the argument of this article that has not arisen previously. Its aim is to investigate the impact of space and time on leadership networks. The theoretically based ABM is calibrated using real data but this only generates hypotheses that the authors claim are subject to empirical validation (but are not actually validated). ${ }^{24}$ From a scientific point of view, this should be acceptable (after all what makes something scientific is that it can be tested not that it has been) but it is an interesting question whether other researchers would be encouraged to try and validate the model in practice. Apart from practical problems in operationalising categories like "convergent leadership", there might seem too great a risk that a theoretically (rather than empirically) derived model just wouldn't survive validation (and despite what anyone says, negative results are much harder to publish). Furthermore, the nature of the derived results (between team leadership declines with spatial separation but is always much higher with "divergent leadership", Sullivan et al., 2015, Figure 5) could be compatible with more than one ABM (the problem of equifinality described above) in the absence of specification.

The overall conclusion from this additional analysis is therefore that, despite its different approach relative to Social Networks, Agent-Based Modelling is neither more common in Network Science nor done substantially differently in terms of calibration and validation.

The argument in this section is detailed but can be summed up by two conclusions. Firstly a typological review of Social Networks and Network Science shows that the concerns raised in our discussion of Hummon's article still clearly apply even after two decades. Even though a few examples of fully calibrated and validated ABMs exist outside the field of network

\footnotetext{
${ }^{24}$ It thus interesting (though the samples are too small to say more) that Social Networks has only one example out of sixteen that is calibrated but not validated while Network Science has two out of three.
} 
analysis, most published applications within the field are neither and almost all of the small number remaining are only validated (with the systematic specification/calibration status of the remainder typically being unclear). ${ }^{25}$ The problems with models that are neither calibrated nor validated or only validated (fitted) therefore remain to be addressed in NS and this analysis thus supports our argument that ABMs following its full methodology still have a distinctive unrealised contribution to make to the scientific understanding of networks (namely the ability to devise scientific tests regarding the outcomes for empirically grounded micro processes). Secondly, despite appearances, actor-based and agent-based approaches are not equivalent and the differences are capable of being clearly articulated and prove to be important. It is not the case that, despite its many merits, the sophisticated work of Snijders et al. (2010) has incorporated the important insights of Agent-Based Modelling into the statistical analysis of networks. Instead, the logic of Agent-Based Modelling methodology makes it clearer what the potential difficulties of actor-based approaches might be. Having cleared the ground and justified the argument, it is now important to be honest about the current limitations of $\mathrm{ABMs}$ and the challenges of realising an effective research programme in the scientific study of networks based on its methodology.

\section{Fulfilling the Potential of Agent-Based Modelling: The Next Steps}

As already mentioned, using ABMs to understand (and respond to) the challenges facing scientific network analysis should not be taken to imply that Agent-Based Modelling has no weaknesses of its own. We have already dealt with one very damaging one, namely the

\footnotetext{
${ }^{25}$ For example, if an ABM is specified "according to theory", how can the reader tell why those particular theories were selected (and other theories or theoretical disagreements disregarded). Supporting such claims is much harder (and less well established methodologically) than justifying the claim that a parameter should have a value of ten (from a survey for example). This example justifies the claim that the specification and calibration status of articles in the sample is often unclear.
} 
dominance (despite excellent examples of calibrated and validated ABMs) of models that are neither calibrated nor validated or only validated. ${ }^{26}$ Furthermore, having a clear vision of what needs to be done in methodological terms (and why) does not of course imply we have yet worked out every detail regarding how to do it. This article is very much programmatic, making clear what the issues are and what might be achieved if systematic attempts were made (in collaboration with social network researchers) to go from logic to implementation. Nonetheless, we can do considerably better than mere exhortation in considering the remaining challenges to the full application of Agent-Based Modelling methodology in the scientific understanding of networks. Many of the ingredients for such a research programme already exist but simply need to be properly organised around the new conception presented here. Firstly, we have claimed that Agent-Based Modelling is interested in empirical grounding for process descriptions while actor-based approaches favour theoretical, technical and fitting justifications for their assumptions. What might that mean substantively? Well, in the context of friendship (to return to the thoroughly analysed Hummon example) it may mean a much greater emphasis on qualitative approaches that are recognised but something of a minority activity in the study of social networks. ${ }^{27}$ (For example, none of examples discussed here make any use of qualitative data.) If you want to know how people make, maintain and think about their friendships then why not ask them to explain in their own words and then build "theory" from patterns in these reports using standard qualitative

\footnotetext{
${ }^{26}$ Such ABMs are so widespread, and calibrated and validated examples so little cited, that it is far too easy to get the wrong impression about what the method is capable of. The absence of calibration and validation may be very widespread but it is nonetheless merely contingent.

${ }^{27}$ But see, for example, the recent excellent discussion by Bellotti (2016).
} 
analysis techniques? ${ }^{28}$ Of course, matters are much less simple than that in detail, but at the moment we are talking about the broad logic of an approach in which subjective accounts (rather than theorised quantitative regularities like a "preference" for homophily) really do lie at the heart of social causation. ${ }^{29}$ How much variation is there in friendship formation processes? How many friendships arise from the kinds of foci discussed by Feld (1981), how many by the action of existing friends (i. e. structurally) and how many through completely idiosyncratic processes (like being marooned at a bus stop or buying a secondhand lawnmower through Facebook from someone two streets away ${ }^{30}$ ) Do friendships formed in these different ways have different likelihoods of persisting? How do friendships develop? What kind of things cause them to grow stronger, weaken or fail? To what extent might geographical separation, time constraints, differences of opinion and life situation or competition from other friendships have these effects? What is the relationship between faceto-face and virtual forms of communication in friendship? Are friends in these spheres viewed distinctly or can electronic communication sustain real world friendships despite geographical separation? What is the role of compulsory and voluntary association in friendship and how do corresponding relationships transition between different levels of

\footnotetext{
${ }^{28}$ Another difficulty is the boundary between what people can consciously articulate and psychological effects that may have to be accessed by other means (like experiments). But this is a general problem, insufficiently attended to, and not limited to Agent-Based Modelling approaches.

${ }^{29}$ There are also institutional issues. For reasons that are not entirely clear qualitative research seems to have been moving away from generalisation and systematic theory building. It may be that qualitative research and Agent-Based Modelling would make a powerful alliance but not in the form in which either kind of research is typically done at present. It will take time and effort to motivate the kind of bridge building needed to address gaps between communities of practice.

${ }^{30}$ Facebook creates interesting new arenas for Feld's conceptualisation. As well as personal friends, who may be distributed globally, we join groups that may be fairly spatially localised (Leicester Cyclists) and thus well connected to face to face interaction or otherwise (Trade Token Collectors).
} 
intimacy? (What distinguishes a colleague who also becomes a friend from one who does not?) Much is known about all these phenomena in a variety of fields and through diverse research methods but there is still a significant challenge regarding how to systematically collate and incorporate such knowledge into ABMs.

A second challenge, faced by ABMs generally and not just those analysing networks, is the creation of agreed standards for progressive research (with associated technical developments). Another drawback of ABMs that are not calibrated and validated is that they must be evaluated on little more than personal taste for the plausibility of their assumptions. This tends to result in fan clubs for particular models with no mechanisms for resolving their divergence in a scientific manner. It is only once we have a significant number of ABMs that are least validated that we can start to talk sensibly about justifying the claim that this model is better than that one. This model is better because it gives better match to real data but it cannot do so by simply having more free parameters to adjust. (For this we need to rigorously define the Agent-Based Modelling equivalent of statistical over fitting - see, for example, Hawkins 2004 - a measure of the intricacy of an ABM relative to the available data.) This model is better than that because, although it only fits equally well, at least some of its parameters are weakly calibrated (or more of its parameters are calibrated). This model is better than that because its specification (the processes it encapsulates) are better empirically supported. This model is better than that because although it only achieves equal fit on an existing set of data, it displays much better fit on another set of data that was not investigated in the original analysis (Chattoe-Brown, 2019). But such a debate is currently foreclosed by an insufficiency of examples (as the typological review above shows). One of the things that collaboration between Agent-Based Modelling and those engaged in the scientific analysis of 
networks should definitely do is make the former community much more mindful of the need for data to distinguish models. ${ }^{31}$

A third issue we have already touched on is clarifying the boundaries between theoretical and empirical model content and the evidential criteria for model content generally. What are the strengths and weaknesses of the concept of "homophily preference" relative to data driven attempts to uncover the actual behavioural processes that give rise to observed degrees and kinds of homophily? Although this clarification task involves challenging conceptual work, it cannot proceed a priori but has to go forward in tandem with collection of relevant data and exploration of associated ABMs.

A fourth issue (which relates to the previous one) is the identification of domain boundaries within which calibration and validation can be systematically implemented. This was, in part, the reason for discussing the work of Bravo et al. (2012) above. Agent-Based Modelling generally assumes that validation takes place at quite a macro level (whole networks for example) but other research designs are possible. For example, agents might be calibrated using laboratory data and then validated on their behaviour in different simulated laboratory experiments that could be compared to their equivalents involving human subjects. Such calibrated agents could then be incorporated into ABMs more broadly with some reasonable hope that they might behave plausibly in other situations. ${ }^{32}$ (This kind of out of sample validation is also a rough and ready antidote to potential over fitting.) Alternatively ABMs might be calibrated to some extent on ego network data and validated on whole networks. The connection to the previous issue is the need to avoid confusion between calibration and

\footnotetext{
${ }^{31}$ Networks also constitute an excellent domain of application for Agent-Based Modelling because their complex structures reduce the chances of fortuitous or non-causal match and, because of excellent prior efforts in the field, there are lots of different ways to characterise networks formally with different information content. ${ }^{32}$ This idea originates with Arthur (1993).
} 
validation. Many laboratory experiments have a rather positivist orientation (looking for patterns in data without subjective accounts). While matching these patterns counts as legitimate validation, the experimental paradigm may have to reduce its positivist tendencies so that relevant calibration data is also accessed. There are research designs to support this like asking people to make decisions in pairs (Dowsett and Burton 2015). This design then allows for recording and analysing discussions as potential qualitative data but this refinement has not, to our knowledge, been applied in Agent-Based Modelling. ${ }^{33}$ Another aspect of this issue is different research designs with regard to the temporal nature of validation. For example, ABMs are often validated against cross-sectional data but, where feasible, out-of-sample validation against time series might be more persuasive.

As can be seen, for each of these issues, Agent-Based Modelling methodology and existing research gives at least some guidance on how to proceed even if all the details are not yet fully worked out. The conclusion of the article overall can now be summed up. Agent-Based Modelling methodology adds to the mere "technical" ability to specify a particular kind of computer programme (which is shared with actor-based approaches). It makes clear how calibration and validation are both needed to derive the full benefits of the approach. If an $\mathrm{ABM}$ is neither calibrated nor validated then we cannot tell what social phenomenon it is supposed to apply to and whether it in fact applies. If it is just validated then the resulting match may not be causal (and more than one ABM may be capable of achieving it). Such ABMs are also much less parsimonious than statistical models, which may further undermine their credibility. It is only if we have reason to believe that the micro behaviour (however approximately) reproduces reality (specification and calibration) that we have any grounds for thinking that a match between real and simulated data might be causal (although we still

\footnotetext{
${ }^{33}$ The problem here may be institutional. Economists and experimental psychologists don't take self-report seriously. Sociologists and social psychologists (who might take it seriously) do very few experiments.
} 
have many technical challenges to solve as regards the extent of match and so on.) In addition, Agent-Based Modelling has other advantages that are relevant to scientific progress in analysing networks. It does not require non-empirical technical assumptions to "make it go" (and thus draws our attention to the potential limitations of approaches that do). It can make use of alternative forms of data (particularly qualitative data) to develop models that directly capture the agency of individual cognition and behaviour (though we are still in the early stages of establishing methodology regarding exactly how to do this - but see, for example, Dilaver, 2015; Ghorbani et al., 2015). This possibility moves us away from the belief that it is unavoidable to use theoretical ideas or conjectural patterns in data as part of a process specification. Finally, this capability (and the challenge of implementing working programmes) gives us a tool to develop and explore a more behaviourally grounded and nuanced view of network processes. ${ }^{34}$ (For example, what might be going on behaviourally in detail behind patterns we observe descriptively like homophily or triad formation?)

The aim of this article has thus been to make it clear enough what the issue is (that AgentBased Modelling has unexploited potential in scientific analysis of networks, what that potential is and why it might be advantageous to exploit it) without attempting to imply that the Agent-Based Modelling approach is therefore "better" than prevailing practices in the analysis of networks (merely different) while at the same time suggesting that a research programme based on these ideas can already start from more than mere agreeable generalities, drawing its strength (as does Agent-Based Modelling itself) from a distinctive and coherent methodology in a developing research field.

\footnotetext{
${ }^{34}$ Another advantage recognised in Agent-Based Modelling is its capability to represent heterogeneity not only in parameters (which is commonplace) but also in things like decision-making (which is not). The existence of a different technology may draw our attention to the fact that assuming a standard (psychological?) pattern for everyone (for example a particular utility function differing only in parameters) is not terribly plausible and probably would not be supported by qualitative data (if that were actually collected).
} 
Funding: This research received no specific grant from any funding agency, commercial or not-for-profit sectors.

Conflicts of Interest: None.

\section{References}

Abdou, M., \& Gilbert, N. (2009). Modelling the emergence and dynamics of social and workplace segregation. Mind and Society, 8(2), 173-191. Arentze, T. A., Kowald, M., \& Axhausen, K. W. (2013). An agent-based random-utility-maximization model to generate social networks with transitivity in geographic space. Social Networks, 35(3), 451-459. Arthur, W. B. (1993). On designing economic agents that behave like human agents. Journal of Evolutionary Economics, 3(1), 1-22. Bavelas, A. (1950). Communication patterns in taskoriented groups. Journal of the Acoustical Society of America, 22(6), 725-730. Bearman, P. S., Moody, J., \& Stovel, K. (2004). Chains of affection: the structure of adolescent romantic and sexual networks. American Journal of Sociology, 110(1), 44-91. Bellotti, E. (2016). Qualitative methods and visualizations in the study of friendship networks. Sociological Research Online, 21(2). Beven, K., \& Freer, J. (2001). Equifinality, data assimilation, and uncertainty estimation in mechanistic modelling of complex environmental systems using the GLUE methodology. Journal of Hydrology, 249(1-4), 11-29. Bravo, G., Squazzoni, F., \& Boero, R. (2012). Trust and partner selection in social networks: an experimentally grounded model. Social Networks, 34(4), 481-492. Chattoe-Brown, E. (2019) Agent based models. In P. Atkinson, S. Delamont, A. Cernat, J. W. Sakshaug \& R. A. Williams (Eds.), Sage Research Methods Foundations. London: Sage. Dilaver, O. (2015). From participants to agents: Grounded simulation as a mixed-method research design. Journal of Artificial 
Societies and Social Simulation, 18(1). Dowsett, A. J., \& Burton, A. M. (2015). Unfamiliar face matching: pairs out-perform individuals and provide a route to training. British Journal of Psychology, 106(3), 433-445. Feld, S. L. (1981). The focused organization of social ties. American Journal of Sociology, 86(5), 1015-1035. Forsyth, E., \& Katz, L. (1946). A matrix approach to the analysis of sociometric data. Sociometry, 9(4), 340-347. Génois, M., Vestergaard, C. L., Fournet, J., Panisson, A., Bonmarin, I., \& Barrat, A. (2015). Data on faceto-face contacts in an office building suggest a low-cost vaccination strategy based on community linkers. Network Science, 3(3), 326-347. Ghorbani, A., Dijkema, G., \& Schrauwen, N. (2015). Structuring qualitative data for agent-based modelling. Journal of Artificial Societies and Social Simulation, 18(1). Gilbert, N., \& Troitzsch, K. G. (2005). Simulation for the Social Scientist (2nd ed.). Milton Keynes: Open University Press. Growiec, K., Growiec, J., \& Kaminski, B. (2018). Social network structure and the trade-off between social utility and economic performance. Social Networks, 55, 31-46. Hägerstrand, T. (1965). A Monte Carlo approach to diffusion. European Journal of Sociology, 6(1), 43-67. Hawkins, D. M. (2004). The problem of overfitting. Journal of Chemical Information and Computer Sciences, 44(1), 1-12. Hendry, D. F. (1995). Dynamic Econometrics. Oxford: Oxford University Press. Henry, A. D., Mitsche, D., \& Prałat, P. (2016). Homophily, influence and the decay of segregation in self-organizing networks. Network Science, 4(1), 81-116. Hummon, N. P. (2000). Utility and dynamic social networks. Social Networks, 22(3), 221-249. Iribarren, J. L., \& Moro, E. (2011). Affinity paths and information diffusion in social networks. Social Networks, 33(2), 134-142. Jackson, M. O., \& Wolinsky, A. (1996). A strategic model of social and economic networks. Journal of Economic Theory, 71(1), 44-74. McGrath, C. A., Vance, C. M., \& Gray, E. R. (2003). With a little help from their friends: Exploring the advice networks of software entrepreneurs. Creativity and Innovation Management, 12(1), 2-10. McPherson, M., Smith-Lovin, L., \& Brashears, M. E. (2006). 
Social isolation in America: Changes in core discussion networks over two decades. American Sociological Review, 71(3), 353-375. Moreno, J. L., Jennings, H. H., \& Stockton, R. (1943). Sociometry in the classroom. Sociometry, 6(4), 425-428. Potashin, R. (1946). A sociometric study of children's friendships. Sociometry, 9(1), 48-70. Ripley, R., \& Snijders, T. A. B., 2009. Manual for SIENA Version 4.0: Provisional Version. Oxford: Department of Statistics and Nuffield College, University of Oxford. Schelling, T. C. (1971). Dynamic models of segregation. Journal of Mathematical Sociology, 1(2), 143-186. Simpson, C. R. (2016). Competition for foundation patronage and the differential effects of prestige on the grant market success of social movement organisations. Social Networks, 46, 29-43. Snijders, T. A. B., van de Bunt, G. G., \& Steglich, C. E. G. (2010). Introduction to stochastic actorbased models for network dynamics. Social Networks, 32(1), 44-60. Stadtfeld, C., Takács, K., \& Vörös, A. (2020). The emergence and stability of groups in social networks. Social Networks, 60, 129-145. Starnini, M., Baronchelli, A., \& Pastor-Satorras, R. (2016). Model reproduces individual, group and collective dynamics of human contact networks. Social Networks, 47, 130-137. Sullivan, S. D., Lungeanu, A., DeChurch, L. A., \& Contractor, N. S. (2015). Space, time, and the development of shared leadership networks in multiteam systems. Network Science, 3(1), 124-155. Watts, D., \& Strogatz, S. (1998). Collective dynamics of "small-world" networks. Nature, 393(6684), 440-442. Will, O. (2009). Resolving a replication that failed: News on the Macy and Sato model. Journal of Artificial Societies and Social Simulation, 12(4). Yang, H.-L., \& Tang, J.-H. (2004). Team structure and team performance in IS development: A social network perspective. Information and Management, 41(3), 335-349. Ziegler, R. (2008). What makes the Kula go round? A simulation model of the spontaneous emergence of a ceremonial exchange system. Social Networks, 30(2), 107-126. 